\title{
Protein networks linking Warburg and reverse Warburg effects to cancer cell metabolism
}

\author{
Dina Johar $^{1}$ (1) | Ahmed O. Elmehrath ${ }^{2}$ () | Rania M. Khalil ${ }^{3}$ () | \\ Mostafa H. Elberry ${ }^{4}$ (ㄴ) | Samy Zaky ${ }^{5}$ (i) | Samy A. Shalabi ${ }^{6,7}$ | \\ Larry H. Bernstein ${ }^{8,9}$ ๑
}

${ }^{1}$ Department of Biochemistry and Nutrition, Faculty of Women for Arts, Sciences and Education, Ain Shams University, Heliopolis, Cairo, Egypt

${ }^{2}$ Faculty of Medicine, Cairo University, Cairo, Egypt

${ }^{3}$ Department of Biochemistry, Pharmacy College, Delta University for Science and Technology, Gamasa, Egypt

${ }^{4}$ Virology and Immunology Unit, Cancer Biology Department, National Cancer Institute, Cairo University, Cairo, Egypt

${ }^{5}$ Hepatogastroenterology and Infectious Diseases, Faculty of Medicine, Al-Azhar University, Cairo, Egypt

${ }^{6}$ Pathology Department, Faculty of Medicine, Cairo University, Cairo, Egypt ${ }^{7}$ Consultant Pathologist, Kuwait, Kuwait

${ }^{8}$ Emeritus Prof. Department of Pathology, Yale University, Connecticut, USA

${ }^{9}$ Triplex Consulting Pharmaceuticals, 54 Firethorn Lane Northampton, MA 01060, USA

\begin{abstract}
It was 80 years after the Otto Warburg discovery of aerobic glycolysis, a major hallmark in the understanding of cancer. The Warburg effect is the preference of cancer cell for glycolysis that produces lactate even when sufficient oxygen is provided. "reverse Warburg effect" refers to the interstitial tissue communications with adjacent epithelium, that in the process of carcinogenesis, is needed to be explored. Among these cell-cell communications, the contact between epithelial cells; between epithelial cells and matrix; and between fibroblasts and inflammatory cells in the underlying matrix. Cancer involves dysregulation of Warburg and reverse Warburg cellular metabolic pathways. How these gene and protein-based regulatory mechanisms have functioned has been the basis for this review. The importance of the Warburg in oxidative phosphorylation suppression, with increased glycolysis in cancer growth and proliferation is emphasized. Studies that are directed at pathways that would be expected to shift cell metabolism to an increased oxidation and to a decrease in glycolysis are emphasized. Key enzymes required for oxidative phosphorylation, and affect the inhibition of fatty acid metabolism and glutamine dependence are conferred. The findings are of special interest to cancer pharmacotherapy. Studies described in this review are concerned with the
\end{abstract}

\footnotetext{
Abbreviations: AMP, Adenosine monophosphate; AFR, ATP flux ratio; AMPK, AMP-activated protein kinase; ATP, adenosine triphosphate; AICAR, 5-aminoimidazole-4-carboxamide-1-D-ribo-furanoside; KG, -ketoglutarate; AD, Alzheimer's disease; 3BP, 3-bromopyruvate; DNP, 2,4-dinitrophenol; B-HOB, $\beta$-hydroxybutyrate; CAV-1, caveolin-1; CBP, cAMP-response element-binding protein; CMA, chaperone-mediated autophagy; CD147, cluster of differentiation; CLL, chronic lymphocytic leukemia; CCOx, cytochrome c oxidase; COX II, cytochrome c oxidase subunit II; DBD, deaminasebinding domain; dbc-AMP, dibutyryl cyclic AMP; EMT, epithelial-mesenchymal transition; ERS, endoplasmic reticulum stress; EV, extracellular vesicle; GAPDH, glyceraldehyde-3-phosphate dehydrogenase; GLS 1, glutaminase 1; GPCRs, G protein-coupled receptors; HK, hexokinase; FDG-PET, 18 fluoro-deoxyglucose-based positron emission tomography; FOXC2, forkhead box protein $\mathrm{C} 2$; FH, fumarate hydratase; GABA, $\gamma$-aminobutyric acid; IMM, inner mitochondrial membrane; IMS, intermembrane space; FeS, iron sulfate; ISC, iron-sulfur clusters; LDHA, lactate dehydrogenase A; LPS, lipopolysaccharide; mTORC1, mammalian target of rapamycin complex 1; mtDNA, mitochondrial DNA; MPC, mitochondrial pyruvate carrier; MAPKs, mitogen-activated protein kinases; mAbs, monoclonal antibodies; NPBac, nodal point of bifurcation anabolic and catabolic processes; OMM, outer mitochondrial membrane; NSCLC, non-small cell lung cancer; OXPHOS, oxidative phosphorylation; PFK, phosphofructokinase; PFK-1, phosphofructose-1-kinase; PFK2, phosphofructo-2 kinase; PGK-1, phosphoglycerate kinase 1; Pi, inorganic phosphate; PI3K, phosphoinositide 3-kinase; p-PKM2, phospho-pyruvate kinase M2; Kv, potassium channels; PC, pyruvate carboxylase; PK, pyruvate kinase; PKM1, pyruvate kinase M1; PKM2, pyruvate kinase M2 ; Rbx1, RING box protein 1; S1P, sphingosine 1-phosphate; SDH, succinate dehydrogenase; TCR, T-cell receptor; TEAD, TEA domain; TAMs, tumor-associated macrophages; Tyk2, Jak1, Jak2), Jak tyrosine kinases; UP1, uncoupling protein 1; UPR, unfolded protein response; YAP, yes association protein; TAZ, WW domain-containing transcription factor.
} 


\section{Correspondence}

Dina Johar, Department of Biochemistry and Nutrition, Faculty of Women for Arts, Sciences and Education, Ain Shams University, Heliopolis, Cairo, Egypt. Email: dinajohar@gmail.com effects of therapeutic modalities that are intimately related to the Warburg effect. These interactions described may be helpful as adjuvant therapy in controlling the process of proliferation and metastasis.

\section{K E Y W O R D S}

aerobic, anaerobic, cancer, glycolysis, metastasis, proliferation, tumorigenesis, Warburg

\section{1 | INTRODUCTION}

The emergence of understanding cancer deregulated molecular mechanisms, and the mechanism for aggressiveness and metastatic potential was a landmark development by Otto Warburg (Nobel Prize, 1931). Such discovery was based on the relationship between lactic acid production from glycogen and lack of oxygen that was elaborated on by Warburg, Meyerhoff and Archibald Vivian Hill in studies of the heat production in skeletal muscle (Nobel Prize, 1922). The PasteurMeyerhof effect extends the theory that less glycogen is consumed in the presence of oxygen than in its absence. It was not until 1932 that an association was made between ATP hydrolysis and muscle contraction. In the subsequent 5 years, a detailed proposal for the reaction sequences was constructed by Embden, Meyerhof, Warburg, Parnas, Neuberg, and the Cori's. This was followed by Warburg's finding that low oxygen is characteristic of cancer cells. ${ }^{1}$

Glycolysis yields only two net molecules of ATP per glucose molecule converted to lactic acid. ${ }^{2}$ Further, the propensity for metastasis is related to the extent of impairment of respiration, which was studied by Hans Krebs in Warburg's laboratory using tissue slices and manometry under conditions of which no heat was lost or gained from the cell growth environment. In sharp contrast to ATP production from most normal tissues, ${ }^{3}$ it was shown by Warburg that cancers frequently develop a modified sugar (glucose) metabolism, whereby a significant portion of the blood sugar consumed by the tumor is converted to one more intermediate beyond pyruvate, that is, to lactic acid, even when oxygen is abundant "Warburg effect." Thus, cancer cells remodel their glycolytic and mitochondrial machinery such that the former is upregulated and the latter is downregulated. ${ }^{4,5}$ Despite these fundamental observations, there are significant inconsistencies in the hypothesis as originally expressed, and it has now become clear that there are a number of factors, ${ }^{6}$ enzymes, ${ }^{7-9}$ and specific isoforms ${ }^{10,11}$ within the context of the "reverse Warburg effect"12 adjacent stromal tissues; cancer associated fibroblasts (CAFs); a Crabtree effect, reactive oxygen species (ROS), activation of transcription factors, ${ }^{13}$ microRNAs ${ }^{14}$ and uncoupling proteins.

\section{1 | Evidence for the Warburg and reverse Warburg effects}

The extent of the Warburg effect in humans tumor NCI60 cell lines screens has been determined by the ratio of glycolytic-to-oxidative ATP flux (AFR), which is found to be highly positively associated with cancer cell migration. ${ }^{6}$ These investigators found that reducing the AFR that may specifically inhibit cancer migration, is achieved by targeting genes that mitigate the Warburg effect. They tested the antimigratory effects of silencing 17 genes in four breast and lung cancer cell lines, and found that up to 13 predictions significantly attenuated cell migration only, either in one or all cell lines, but had no effect on cell proliferation. These investigators found a high correlation between measured and predicted lactate secretion levels across various conditions of oxygen supply by systematically assessing the extent of lactate secretion across wide range of cancer cell lines. They were able to determine the ratio of glycolytic versus oxidative capacity in a cell using its extracellular acidification rate (ECAR, a proxy of lactate secretion) and its oxygen consumption rate (OCR). They found in comparing the ratio between the AFR in glycolysis versus AFR in oxidative phosphorylation, that higher AFR values denoted more Warburg metabolic profile. Further, the ratio between glycolytic and oxidative AFR is significantly associated with cancer migration.

Another study ${ }^{14}$ integrated the genome-scale metabolic model of hepatocytes and mouse experimental data with germline deletion of MiR122a (MiR122a $\pm / \pm$ ) to infer Warburg-like effects. The results found elevated expression of MiR-122a target genes in MiR122a $\pm / \pm$ mice, in particular, those encoding for metabolic enzymes, by analyzing the flux distributions of the genome-scale metabolic model in normal and deficient states. ${ }^{14}$ They compared the flux fold change of the genome-scale metabolic model computational results with metabolomic profiling data and reported that the Dopa decarboxylase Ddc gene demonstrated the highest similarity ratio of $95 \%$ to the biological hypothesis of the Warburg effect, and similarity of $75 \%$ to the experimental observation.

The Warburg effect has been regarded as a consequence of an imbalance between maximum rates of 
glycolysis and pyruvate oxidation. It has been proposed that glucose uptake is facilitated by the transport of glucose over a plasma membrane, which requires a glucose transporter/solute carrier (GLUT/SLC2A) family. ${ }^{4}$ Cancer cells in a hypoxic environment overexpress GLUTs, especially GLUT1 and GLUT3. Since GLUT1 and GLUT3 have a high affinity for glucose, cancer cells can efficiently consume the available glucose, thereby reducing the glucose concentration in the tumor microenvironment. In addition, the same hypoxia causes HIF-containing HIF- $1 \alpha$ and HIF-1 $\beta$ subunits overexpressed. $^{15}$

\section{2 | Cell-cell communication and the reverse Warburg effect}

The concept of a "reverse Warburg effect" implies that aerobic glycolysis may take place in the fibroblastic tumor stromal compartment, rather than in the epithelial cancer cells themselves. These CAFs then undergo myofibroblastic differentiation, and secrete lactate and pyruvate. Through cell-cell communication, epithelial cancer cells could then take up these energy-rich metabolites, into the mitochondrial Krebs cycle, promote ATP generation, resulting in a higher proliferative capacity. In such way, the reverse Warburg effect is an alternative model to the "ancestor mutant model," as the epithelial cancer cells instruct the stromal cells to transform into a woundhealing stroma, by providing the energy-rich microenvironment for facilitating tumor growth and angiogenesis. ${ }^{12}$

Transfer of pyruvate/lactate from myofibroblasts to epithelial cancer cells and endothelial stroma occurs via monocaroboxylate transporters such as MCT1/4. In this scenario, CAFs and the tumor epithelial cells would be metabolically coupled. ${ }^{4}$ The overexpressed proton-linked MCT113 and MCT4 drive lactic acid secretion from the cytoplasm into the extracellular fluid. ${ }^{3}$ The $\mathrm{T}$ cells generate more lactic acid into the microenvironment, and a lower $\mathrm{pH}$ and higher lactic acid concentration caused by tumor cells, in turn, inhibits lactic acid secretion from $\mathrm{T}$ cells. The lactic acid inhibits the innate immune response by downregulating lipopolysaccharide (LPS)induced genes, which delays LPS-induced phosphorylation of serine/threonine kinase 1 (AKT) and the degradation of I-kappa-B (IkB). As a result, immune cells invasion is limited, contributing to tumor immune evasion. In this environment, a "reverse Warburg effect" is driven by the cancer cells secreting hydrogen peroxide to create a "pseudo-hypoxic" environment that activates HIF-1 $\alpha$, glycolysis, and MCT4 expression of the stromal cells.

\section{3 | Complex metabolic dynamics contributing to the Warburg and the reverse Warburg effects}

Investigators proposed that the complex temporal and spatial dynamics of intracellular ATP demand drive cellular metabolism to provide energy for constant baseline needs but also maintain capacity to rapidly respond to steep fluxes in demand of the tumor. Short-term fluxes in demand of ATP including division, migration, and invasion, which requires increased activity of membrane transporters, that is necessary to maintain functions inherent in the malignant phenotype, associated with the gradient increase in substrate and cycles of normoxia and hypoxia. Long-term dynamics requires maintaining high metabolic capacity to meet uncommon spikes in demand and maintain the cell's optimal merge of glycolytic and oxidative capacity. Others have constructed a regulatory network of genes and metabolites from which a core circuit containing HIF-1, AMPK, and ROS were extracted. ${ }^{13}$ They concluded that while normal cells have an oxidative state and a glycolytic state, cancer cells can access a hybrid state with both metabolic modes coexisting. The cancer cells had a high ROS production and/or oncogene activation, such as Rat sarcoma (RAS), Myelocytomatosis-related family of transcription factors (MYC), and cellular sarcoma Proto-oncogene tyrosineprotein kinase (c-SRC). They developed two models using AMP-activated protein kinase (AMPK) and HIF-1 downstream genes to quantify the activity of glycolysis and oxidative phosphorylation. They applied the AMPK and HIF-1 signatures to the Cancer Genome Atlas patient transcriptomics data of multiple cancer types and singlecell RNA-seq data of lung adenocarcinoma. They reported a reverse correlation between AMPK and HIF-1 activities and the association of metabolic states with oncogenes and concluded that the hybrid phenotype contributes to metabolic plasticity. This hybrid phenotype allows cancer cells to adapt to various microenvironments to favor tumorigenesis and metastasis. They propose a new cancer therapeutic strategy by targeting the hybrid state. To learn more about cancer, we need to know more about metabolic pathways incorporated in cancer mechanism and contributes to the Warburg and the Reverse Warburg, we elaborate on such pathways in the following sections.

\subsection{Altered regulation of Warburg by glycolytic enzymes and their isoforms}

Abnormal differential expression in enzymatic activity is reported in Warburg effects such as overstimulation of all 
glycolytic enzymes, especially with respect to the ratelimiting enzymes hexokinase (HK), phosphofructokinase (PFK), and pyruvate kinase (PK). Further, upregulation of glutaminase and pyruvate carboxylase (PC) along with inactivation of fumarase and mutations in succinate dehydrogenase (SDH) were reported. ${ }^{16,17}$

Cancer cells exploit isoforms of glycolytic enzymes to enforce the Warburg effect. In that sense, Warburg is an effect of cancer. The splicing switch to the M2 isoform of pyruvate kinase (PKM2) is an example. ${ }^{5}$ Some studies show crosstalk between the degree of malignancy progression and overactivation of $\mathrm{PK}^{7,15}$ In humans, ectopic expression of PKM1 isoenzymeinduced PK arrests cell proliferation and results in discrete changes in metabolism that are limited to nucleotide production. ${ }^{10}$ PKM2 is strongly connected to proliferation, it is expressed in the development of embryos, adult tissues, and cancer cells. Unfortunately, a complete picture of how PKM2 benefits proliferating cells has yet to be clarified, as it is an interesting point in the Warburg-based cancer mechanism. ${ }^{8}$ Another example of isoforms of glycolytic enzymes that has been specifically associated with cancer is isocitrate dehydrogenase (IDH2). Of great importance is the repeated observation that lactate dehydrogenase isoenzyme A decreases in many cancers. The predominantly $\mathrm{H}$-type $\mathrm{LDH}$ is associated with inhibition by pyruvate, inhibiting the reaction as it binds with the NAD+ formed and the $\mathrm{LDH}$, more favorable to a shift to glycolysis.

\section{5 | Pathways of anaplerosis and cell proliferation mediated by $\mathrm{PC}$ and glutaminase 1 contribute to the reverse Warburg effect}

In normal cells, the chief anaplerotic enzyme contributing to the reverse Warburg is PC. ${ }^{16}$ An increase in the level of PC was reported in some tumor cell lines, ${ }^{18}$ and perfused rat livers showed a high alanine flux resulting from PC activity. ${ }^{19}$ Further, glutaminase converts glutamine to glutamate, to be used by Krebs cycle. ${ }^{11}$ Different types of cancer cells have different requirements for pathways of anaplerosis and cell proliferation mediated by PC and glutaminase 1 (GLS1). Further, it has been found that tumor survival and propagation require PC-mediated anaplerosis in early-stage non-small cell lung cancer (NSCLC). ${ }^{9,16}$

Myc plays a role in stimulating glutamine catabolism, which includes the repression of miR-23a and miR-23b. This adds a level of complexity in tumor metabolism that involves the metabolic relationship between hypoxic and nonhypoxic regions of tumors including the stroma. This suggests reassessment of cancer metabolism to consider glutamine catabolism to effectively target in therapy. ${ }^{5}$

Glutamate is released from activated synapses and taken up by astrocytes triggering an increase in glycolysis and lactate production. ${ }^{20}$ Lactate can be oxidized by the neurons in response to their increased energy requirement to produce ATP. In the proposed reverse Warburg effect, hydrogen peroxide is secreted by cancer cells leading to oxidative stress in the associated fibroblasts. The resulting loss of mitochondrial function acts as a switch from aerobic metabolism to glycolysis, with lactate as the end product. Lactate is capable of replacing glucose as an energy source, and it is capable of increasing vascular endothelial growth factor expression. The fibroblasttumor metabolic coupling in the reverse Warburg effect is analogous to the metabolic symbiosis seen in the brain. $^{20}$

\section{I IRON DEFECT REGULATION OF BIOGENESIS}

Iron $(\mathrm{Fe})$ is a vital mineral for living cells ${ }^{21}$ due to its important role in oxygen transport and oxidative phosphorylation beside heme, heme enzymes, and proteins. ${ }^{22,23}$ A defect in mitochondrial DNA (mtDNA) leads to loss of stability of the nuclear genome, which leads to cellular crisis; it is caused by a decrease in the mitochondrial membrane potential rather than the absence of respiration. A defect in the iron-sulfur clusters (ISC) biogenesis has been identified in the cell crisis. In addition, it has been found that downregulation of the nonmitochondrial ISC protein biogenesis is enough to cause greater genomic instability in cells with normal mitochondrial function. ${ }^{24}$ Therefore, dysfunction of mitochondria arouses nuclear genome instability by impeding the ISC-containing protein(s) production. ${ }^{19}$ Another possible cause of disturbed iron metabolism is a defect in Frataxin protein, which is an inner mitochondrial membrane, and mitochondrial matrix protein that plays a role in iron synthesis and storage. ${ }^{24}$ Frataxin defect may be a possible problem that plays a vital role in ISC and heme synthesis. Frataxin deficiency results in the mitochondrial iron loading. ${ }^{25}$ Some cells go into cellular crisis due to nuclear genomic instability as a result of mtDNA loss. This was associated with disturbed mitochondrial iron metabolism. Iron is a vital mineral in activation of ribonucleotide reductase, which is key enzyme in DNA synthesis; therefore, iron is needed for cellular proliferation. Correction of alkalosis in case of mitochondrial failure may be an effective approach as intracellular alkalosis 
stimulates mitosis that is not affected by inhibitory signals. $^{26}$

\section{1 | Tumor suppression gene TP53 links to Warburg}

Normally, p53-mediated inhibition of phosphofructokinase (PFK) promotes ATP synthesis through enhancing oxidative phosphorylation and suppression of glycolysis. P53-induced enhancement of oxidative phosphorylation comes from expression of the gene that encodes cytochrome c oxidase (CCOx), which is important in mitochondrial cytochrome c oxidase complex. TP53-inducible glycolysis and apoptosis regulator (TIGAR) is a P53 gene/ protein that inhibits glycolysis by downregulating cellular fructose-2,6-bisphosphate: the latter is an enhancer of PFK1. ${ }^{27,28}$ Because Warburg effect increases lactic acid, any abnormality in lactate production and signaling will lead to tumorigenesis. ${ }^{29}$ In that perspective, Warburg can be thought of as a cause of cancer. One of the most frequently mutated genes in cancers, p53, modulates the balance between the utilization of respiratory and glycolytic pathways, increasing glucose utilization for lactagenesis and lactate exchange among cells. ${ }^{27}$ These authors suggest that in glycolytic cancers, increased glycolysis is chronic which may lead to increased proteolysis for gluconeogenesis, as well as for glutaminolysis to increase cytosolic pyruvate for lactate production. Chronic increased proteolysis for gluconeogenesis and glutaminolysis could explain cachexia in cancer.

Oncogenes-mediated anablerosis functions in cancer cells promote nodal point of bifurcation of anabolic and catabolic processes (NPBac) and inhibit both catabolism in cancer cells and degradation of cadherin-catenin complexes. This prevents adhesion of cancer cell membranes, ${ }^{30}$ and deploys the Warburg effect. ${ }^{26} \mathrm{CD} 147$, a glycoprotein on cell membranes of cancer cells, plays a vital role in tumorigenesis, cellular death, migration, and differentiation. ${ }^{31,32}$ CD147 modulation of Warburg effect comes from its continuous stimulation of glycolysis and its inhibition of oxidative phosphorylation. ${ }^{33}$

\section{2 | Hypoxia inducible factor-1 $\alpha$ induces strong Warburg phenotype in cancer cell metabolism}

HIF-1 $\alpha$ induces strong Warburg phenotype via affecting glucose and pyruvate metabolism and mitochondrial biogenesis. ${ }^{34-38}$ HIF-1 $\alpha$ plays a critical role in shifting cancer cell metabolism from oxidative phosphorylation to aerobic glycolysis. ${ }^{34,37}$ This function has been traced back to the oxygen responsive regulatory HIF-1 $\alpha$ subunit. Overexpression of HIF- $1 \alpha$ is common in many types of solid tumors including those of the stomach, colon, ovary, lung, breast, pancreas, and kidney. ${ }^{35}$ The dominant mode of HIF- $1 \alpha$ regulation is through oxygen-dependent abolishing of HIF- $1 \alpha$ subunits, Figure 1 . In cancer, other several oxygen-independent mechanisms are involved in HIF- $1 \alpha$ activation including increasing the level of reactive oxygen species (ROS) stress, ${ }^{39,40}$ mutations leading to inactivation of tumor suppressors, for example, phosphatase and tensin homolog (PTEN), ${ }^{41} \mathrm{p} 53,{ }^{42} \mathrm{pVHL}^{43}$ or activation of oncogene factors as cMyc, ${ }^{44} \mathrm{Ras}^{45}$ or pathways as PI3K/AKT. ${ }^{46}$ Accumulation of tricarboxylic acid metabolites as succinate and fumarate as a result of mutant dysfunctional succinate dehydrogenase and fumarate hydratase, respectively, has been reported as an underlying cause of HIF-1 stabilization in congenital cancer types. ${ }^{47,48}$ The two metabolites compete with $\alpha$-ketoglutarate for binding to PHD2, thereby decreasing ubiquitinylation, hydroxylation, and proteasomal degradation of HIF- $1 \alpha{ }^{47,48}$ Lactate and pyruvate levels, the end products of glycolysis, regulate the expression of hypoxia inducible genes independent of hypoxic stimulation. ${ }^{49}$ It has been postulated that pyruvate plays a possible inhibitory action against HIF-1 $\alpha$ degradation at the steps involving proline hydroxylation, pVHL tumor suppressor protein binding or ubiquitin conjugation. It is therefore shown that the process of glycolysis is able to cause activation of HIF-1 DNA binding activity leading to the enhanced expression of several HIF-1- activated genes. $^{50}$

HIF-1 $\alpha$ triggers the expression of glucose transporters (GLUT1 and GLUT 3) and their translocation to the plasma membrane activating glucose uptake., ${ }^{35,38}$ As it activates the expression of all glycolysis enzymes converting glucose to pyruvate, especially those thought of as flux- limiting in the metabolic pathway (HK1 and HK2), (PK, the embryonic isoform M2), and PFK. ${ }^{35,38}$ HIF-1 $\alpha$ upregulates lactate dehydrogenase A (LDHA), which transforms pyruvate to lactate, ${ }^{51}$ and monocarboxylate transporter 4 (MCT4), which transports lactate out of the cell. ${ }^{52}$ In addition, HIF- $1 \alpha$ decreases the transformation of pyruvate to acetyl-CoA by pyruvate dehydrogenase (PDH) decreasing influx into the Krebs cycle and the subsequent mitochondrial oxidative phosphorylation. Thus, HIF-1 $\alpha$ activates the PDH kinase 1 (PDK1) gene, which inhibits PDH. ${ }^{53,54}$ HIF- $1 \alpha$ impedes mitochondrial biogenesis and cell respiration through depressing c-Myc activity and promoting its proteosomal degradation in VHL-deficient renal cell carcinoma. ${ }^{55}$ In contrast, HIF$1 \alpha$ interacts with c-Myc to stimulate aerobic glycolysis by inducing HK2 and PDK1. ${ }^{56}$ Studies reported heart- 


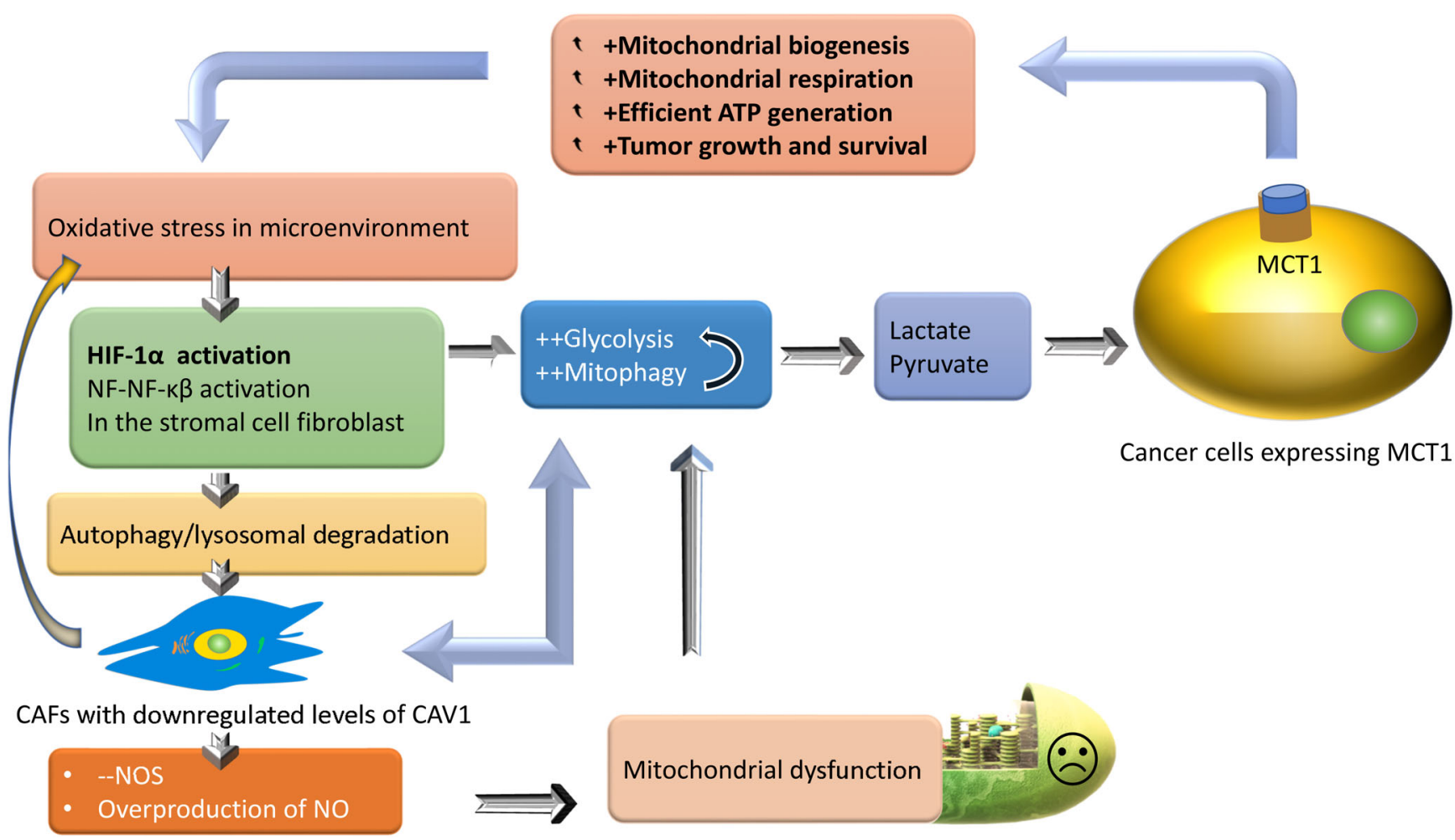

F I G U RE 1 Warburg and the reverse Warburg phenomena. It shows metabolic coupling between glycolytic stroma fibroblast and cancer cells. Cancer cells induce CAFs phenotype that feed tumor cells with cycled nutrients for oxidative phosphorylation and tumor survival via oxidative stress and HIF- $1 \alpha$ activation. Under normoxic conditions, HIF- $1 \alpha$ is hydroxylated at conserved proline residues by prolyl hydroxylase domain protein 2 (PHD2) using $\mathrm{O} 2$ and $\alpha$ - ketoglutarate as co-substrates. Hydroxylated HIF- $1 \alpha$ is bound by the vonHippelLindau tumor suppressor protein (pVHL), then ubiquitinylated by an E3 ligase complex containing elongin B, elongin C, cullin 2 and RING box protein 1 (Rbx1) for final proteosomal degradation. ${ }^{57,58}$ Additionally, oxygen availability promotes hydroxylation of HIF-1 $\alpha$ on the asparagine 803 residue via factor inhibiting HIF-1, an $\alpha$-ketoglutarate-dependent dioxygenase. This blocks interaction of HIF-1 with the coactivators; histone acetyltransferase p300 (p300) and cAMP-response element-binding protein (CBP) inhibiting its transcriptional action. ${ }^{59}$ Under hypoxic conditions, HIF- $1 \alpha$ binding to pVHL is inhibited because prolyl hydroxylation is reduced, leading to HIF-1 $\alpha$ stabilization and dimerization with HIF-1 $\beta$. The stable complex then recruits the coactivators and binds to the hypoxia response element (HRE) site of target genes resulting in their transcriptional activation. ${ }^{35}$ HIF-1 plays key role in the reprogramming of cancer metabolism by activating transcription of genes encoding glucose transporters and glycolytic enzymes, which take up glucose and convert it to lactate; pyruvate dehydrogenase kinase 1, which shunts pyruvate away from the mitochondria; and BCL2 and adenovirus E1B 19-kDa-interacting protein3 (BNIP3), which triggers selective mitochondrial autophagy, decreased respiration and promoted cell survival under prolonged hypoxia. ${ }^{35,37}$ The shift from oxidative to glycolytic metabolism allows maintenance of redox homeostasis and cell survival under conditions of prolonged hypoxia. Many metabolic abnormalities in cancer cells increase HIF-1 $\alpha$ activity. As a result, a feed-forward mechanism can be activated that drives HIF- $1 \alpha$ activation and may promote tumor progression

anchoring cells (HANC), which acquired cetuximab, an EGFI-receptor-blocking monoclonal antibody, resistance, had high levels of HIF-1 $\alpha$ expression and were highly glycolytic. ${ }^{60}$ Cancer therapy has been proposed by interrupting the expression and/or activity of HIF$1 \alpha .^{36}$ For example, cetuximab was able to inhibit glycolysis and proliferation in cetuximab-sensitive squamous cell carcinoma (HNSCC) cells in head and neck. Such effects were mediated via downregulating HIF-1 $\alpha$ subunit through blocking downstream EGFI receptor signaling.

\section{3 | Mitochondrial uncoupling protein UCP2 links Warburg to cancer cell metabolism}

One of the mitochondrial alterations underlying the metabolic shift of cancer cells from oxidative phosphorylation to aerobic glycolysis is mitochondrial uncoupling. ${ }^{61,62}$ Uncoupling of mitochondrial respiration from phosphorylation of ADP into ATP is mediated via uncoupling proteins. ${ }^{63}$ One of them is UCP2, which is anion/metabolite carrier located in the inner mitochondrial membrane and 
involved in modulation of cancer cell energy balance via mediating a number of sequential events. ${ }^{63,64}$ Among such events are proton leak/conductance from the intermembrane space to the mitochondrial matrix; bypassing ATP synthase; inhibiting the proton motive force from being excessive; reducing inner mitochondrial membrane potential; shifting metabolism to the use of non-glucose carbon sources (as fatty acids and glutamine) to maintain mitochondrial function; reducing ROS generation, and eventually providing survival and oxidative-stress protective advantages for cancer cells. ${ }^{62,64,65}$ Overexpression of UCP2 was found in breast, leukemia, ovarian, bladder, esophagus, testicular, colorectal, kidney, pancreatic, lung, and prostate tumors. ${ }^{66}$

Evidence demonstrates a link between UCP2 and the Warburg effect. ${ }^{61,66-70}$ HCT116 colon cancer cells overexpressing UCP2 produced more lactate than control cells, indicating higher rates of glycolysis. ${ }^{67}$ Studies $^{61} \mathrm{rev}$ ealed that the exposure of OCI-AML3 leukemia cells to mesenchymal stromal cells induced UCP2 expression in cancer cells. That was associated with accumulation of lactate in the culture medium, indicative of the Warburg effect and siRNA-mediated inhibition of UCP2 in the leukemia cells resulted in decreased lactate production. ${ }^{61}$ Ayyasamy and his colleagues created a cellular model of the Warburg effect by developing an epithelial cell line lacking mitochondrial DNA termed (rho(0). ${ }^{6,71}$ Among the regulated genes, UCP2 expression was predominantly higher in rho(0) cells suggesting that UCP2 may inhibit ROS accumulation induced by mitochondrial defects linked to Warburg effect. UCP2 has been proposed to function as a uniporter for pyruvate, ${ }^{72}$ facilitating pyruvate efflux from mitochondria restricting glucose availability for respiration. ${ }^{73}$ Furthermore, UCP2 catalyzes the export of $\mathrm{C} 4$ metabolites, particularly oxaloacetate, out of mitochondria at the expense of phosphate by an $\mathrm{H}^{+}$. assisted mechanism. The low availability of oxaloacetate regulates the entry of acetyl-CoA into the Krebs cycle. ${ }^{74}$ Another group confirmed the pro-glycolytic effect of UCP2 demonstrating that UCP2 can inhibit the oxidation of the glycolytic enzyme glyceraldehyde 3-phosphate dehydrogenase (GAPDH) in the cytoplasm of cancer cells and its subsequent translocation to cell nuclei, ${ }^{68}$ where the enzyme favors transcriptional induction of cell deathrelated genes. ${ }^{75}$ It has been demonstrated that ectopic expression of UCP2 in pancreas cancer cells sustained the metabolic shift from mitochondrial oxidative phosphorylation to glycolysis and that sensitized the cells to the treatment with the glycolytic inhibitor 2-deoxy-D-glucose. The molecular mechanism underlying such findings was UCP2-induced expression of hnRNPA2/B1, which is involved in the regulation of both GLUT1 and PKM2 mRNAs, and of LDH increasing the secretion of L-lactic acid. ${ }^{69}$ More recently, using the skin cell transformation JB6 model, it has been demonstrated that UCP2 overexpression induce AKT-dependent activation of PFK2 or fructose-2, 6-bisphosphatase 2 (PFKFB2), a key regulator of glycolysis. Upregulation of PFKFB2 expression correlated with enhanced glucose uptake and elevated fructose 2, 6-bisphosphate (Fru-2, 6-P2) levels, PFK-1 activity and lactate production, that is, promoted metabolic shift to glycolysis "the Warburg effect". ${ }^{70}$ Altogether, the data identify UCP2 as a potential molecular target in cancer. ${ }^{67}$ Genipin is a natural dietary compound used as a potential anticancer agent. It acts by interfering with UCP2 function to dissipate energy and restrict ROS production through proton leakage. ${ }^{66,76}$

\section{4 | Caveolin-1 in cancer cell metabolism}

Caveolin-1 (CAV1), an integral membrane protein associated with endocytosis, cell migration, cholesterol distribution, and signal transduction. Research findings suggest that CAV1 is altered in several cancer types. ${ }^{77}$ Several studies reveal that CAV1 is involved in the modulation of glycolytic activities, however, such tumor promotion or suppression roles have been proposed to be context-dependent. ${ }^{78}$ For example, stable expression of CAV1 after exposure to a volatile anesthetic (isoflurane) enabled cancer cells from escaping apoptosis induced by tumor necrosis factor-related apoptosis-inducing ligand (TRAIL). The pro-survival role of CAV1 was mediated by enhanced glycolysis. ${ }^{79}$ CAV1 could provide colorectal cancer cells growth advantages by upregulating GLUT3-mediated glucose uptake and aerobic glycolysis. ${ }^{80}$ CAV1 interacted with low-density lipoprotein receptorrelated protein 6 (LRP6) to generate an integrated signaling module that led to the activation of insulin- and IGF-I receptors (IR/IGF-IR) and resulted in stimulation of Act-mTORC1 signaling and aerobic glycolysis in prostate cancer. Such effects were correlated with upregulating HK2 and GLUT3 expression. ${ }^{81}$ It has been proposed that, in cancer, CAV1 may function as a scaffolding protein for PFK, aldolase (ALD) and perhaps other glycolytic enzymes ${ }^{78}$ in vascular smooth muscle cells, resulting in localization of glycolytic enzymes to plasma membrane and thus membrane-associated glycolysis. ${ }^{82}$ On the other hand, loss of the tumor stromal CAV1 may be a novel biomarker for the Warburg effect. ${ }^{83}$

Studies on the loss of CAV1 expression in luminal breast cancer epithelial cell line (MCF7) revealed activation of NF-E2-related factor 2 (Nrf2): a transcription factor that induces the expression of manganese-superoxide 
dismutase (MnSOD). MnSOD expression led to an increase in the glycolytic rate, dependent on mitochondrial $\mathrm{H}_{2} \mathrm{O}_{2}$ production and the activation of AMPK. ${ }^{84}$ Lack of stromal CAV1 was associated with tumor recurrence, metastasis, and poor clinical outcome in breast cancer. $^{12,85}$ Several glycolytic enzymes, including aldolase A, enolase 1, PGK1, PKM2, and LDHA, were upregulated in bone marrow-derived stromal cells from CAV1 knockout (KO) mice. ${ }^{12}$ Outschoorn et al. developed a co-culture system of breast cancer epithelial cells and immortalized fibroblasts to explain it. In this model, cancer cells induced oxidative stress via ROS overproduction, and autophagy: the processes of processing and recycling mitochondrial contents by lysosomes, in the adjacent fibroblasts. That was associated with downregulation of stromal CAV1, thereby acquiring CAFs phenotype. ${ }^{86}$ Loss of CAV1 elicited further ROS generation and DNA damage in the fibroblasts. Also, it induced mitochondrial dysfunction, and HIF- $1 \alpha$ activation glycolytic switch. To dissect the mechanism underlying decreased mitochondrial mass and enhanced glycolysis in the fibroblast, autophagic destruction of mitochondria (mycophagy) was activated as a consequence of oxidative stress and HIF-1 $\alpha$ activation. It was also demonstrated that nitric oxide (NO) overproduction, secondary to CAV1 loss, caused mitochondrial dysfunction in CAFs via a paracrine signaling induced further CAV1 downexpression. ${ }^{86}$

\section{3 | POTENTIAL THERAPEUTIC STRATEGIES TARGETING THE WARBURG EFFECT}

Cancer cells display extremely versatile aerobic glycolysis, and therefore, it may be logical to enterprise anticancer drugs established on the features and mechanisms of aerobic glycolysis mechanism and on the association between aerobic glycolysis and cancer progression. Lactate transport inhibitors would be predicted to kill cancer-associated fibroblasts via induction of intracellular acidification. Similarly, lactate transport inhibitors would eliminate extracellular source of lactate and pyruvate for epithelial tumor cells leading to their starvation. Energyrich metabolites (as pyruvate and lactate from aerobic glycolysis) were proposed to transfer to the adjacent tumor cells and used to feed mitochondrial biogenesis and respiration for great energy production and promoted proliferation. ${ }^{12,86}$ This metabolic coupling between the supportive glycolytic stromal fibroblasts and oxidative tumor cells could be a potential target for cancer therapy. Cancer therapy should target mitochondrial metabolic dysfunction beside immune system support. Cetuximab is an EGF-I receptor-blocking monoclonal antibody used as antiproliferative agent in many conditions, which downregulates the $\alpha$ subunit of HIF-1. As mentioned earlier in this review, HIF-1 has a critical role in mediating Warburg effect in cancer cells. Certain types of head and neck squamous cell carcinomas (HNSCC) are sensitive to cetuximab as it downregulates LDH $\alpha$ and HIF-1 $\alpha$. Overexpression of HIF- $1 \alpha$ associated with HNSCC resistance tocetuximab may be overcome by knockdown of LDH $\alpha$ expression. ${ }^{60}$ Figure 2 summarizes the potential therapeutic strategies targeting the Warburg effect.

Advances in the field of epigenetics led to discovery of new metabolic changes in cancer cells. Food turnover results in accumulation of certain protein and cofactors that control gene expression through epigenetics. One of the regulators of cellular metabolism is AMPK that acts as a regulator in several pathways that link energetics to longevity. AMPK activation is through high AMP to ATP ratio leading to inhibition of cellular proliferation. The serine/threonine kinase LKB1 is a known tumor suppressor that activates AMPK thus inducing AMPK effects. ${ }^{87}$

A lot of attention has been directed to malignant brain tumors. A novel therapeutic strategy is suggested through metabolically targeting malignant cells. Normal brain cells depend on glucose for energy production however in the extreme conditions, that is, low blood glucose, normal cells shift to ketone bodies $\beta$-hydroxybutyrate ( $\beta$-OHB). Unlike normal brain cells, malignant brain cells cannot shift to ketone bodies as result of genetic mutation. Although lowering blood glucose between 3.0 and $3.5 \mathrm{mM}$ (55-65 mg/dl) and elevating $\beta$-OHB ranges between 4 and $5 \mathrm{mM}$ will affect malignant brain cells regardless their anatomical site and cellular histology leaving brain cells unaffected. $^{88}$ Glioblastoma multiforme (GBM) is one of the most aggressive tumors. Lipoic acid and hydroxycitrate were effective in animal models of GBM. With the additional use of chemotherapy promising results have been shown in glioblastoma, brain metastasis and lung cancer. ${ }^{89}$ A potential therapeutic approach is to induce glioblastoma differentiation. A proposed differentiation model is used to define the mechanism of its differentiation. cAMP activators were used in this model to direct GBM into astroglia. Data showed oxidative phosphorylation and mitochondrial biogenesis are involved in the differentiation process. Dibutyryl cyclic AMP reduces levels of lactate production thus reverses the Warburg effect. CREB-PGC1 $\alpha$ pathway induces metabolic shift and affects mitochondrial biogenesis. Mitochondrial division inhibitor 1 blocks mitochondrial biogenesis through inhibition of PGC1 $\alpha$ leading to inhibition of differentiation. ${ }^{33}$ Xing et al. show that the metabolic shift from glycolysis to oxidative phosphorylation drives differentiation of GBM cells into astrocytes by cAMP activation. Mechanistically, 


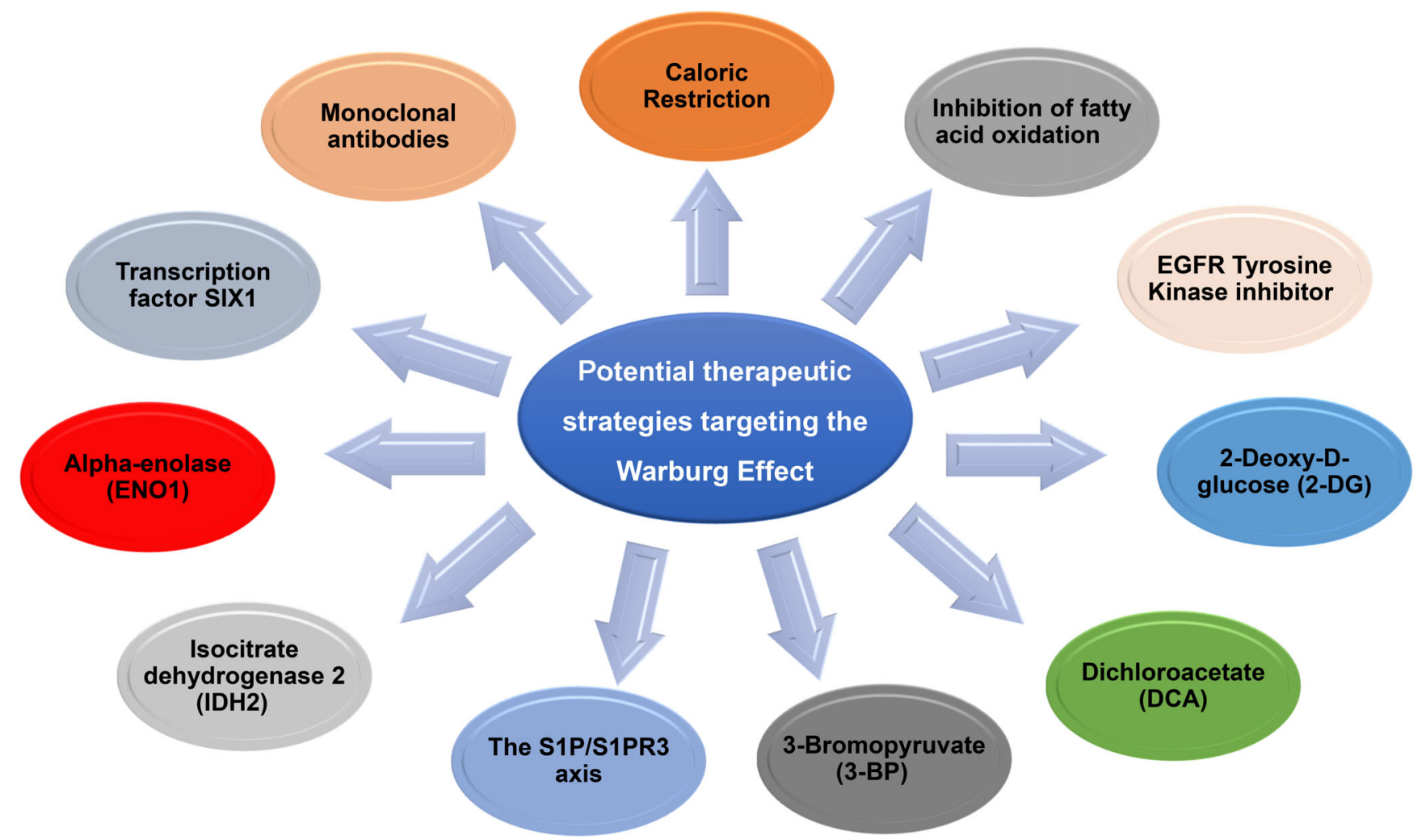

FIG URE 2 Potential therapeutic strategies targeting the Warburg effect

the cAMP-CREB-PGC1 $\alpha$ signal mediates mitochondrial biogenesis, which leads to metabolic reprogramming, induced differentiation, and tumor growth inhibition. ${ }^{90}$ Further, Cyclin G2 is considered as tumor suppression gene in many cancers. Study showed Cyclin G2 downregulates LDHA: an important enzyme in glycolysis in glioma that was expressed in low levels denoting poor prognosis of glioma. ${ }^{91}$

In hepatocellular carcinoma (HCC), CD147 may be a potential target for treatment. ${ }^{32} \mathrm{CD} 147$ is a transmembrane protein that is expressed in HCC cells that regulate cellular proliferation and differentiation. CD147 also promotes the Warburg effect by inhibiting mitochondrial biogenesis and oxidative phosphorylation and promoting glycolysis. $^{92}$

\section{1 | Caloric restriction}

Excess calories can lead to obesity promoting tumorigenesis. Therefore, the opposite might prove to be true. In fact, a number of animal model studies showed that caloric limitation prolongs life span. ${ }^{93,94}$ Moreover, it inhibits tumorigenesis, possibly by reduced IGF-1 levels. Tumors with activating PI3K mutations resist caloric restriction, suggesting that caloric restriction affects growth factor- receptor tyrosine kinase signaling by reducing IGF-1 levels and eventually mutations that activate the PI3K pathway make tumor cells resistant tocaloric restriction. ${ }^{95}$ The basis of reduced tumorigenesis due to caloric restriction might be related to autophagy and mitophagy. Such processes are enhanced by AMPK activation in a reduced energy state, ${ }^{96}$ in turn, reduces mTOR activity and increases sirtuin activity, ${ }^{97,98}$ reducing cell growth. ${ }^{99}$ Inhibition of autophagy stimulates gradual cell deterioration. This could be attributed to the inability of cells to get rid of faulty mitochondria, leading to increased oxidative stress and aging. ${ }^{100,101}$ However, caloric restriction enhances mitophagy, which helps cells to get rid of the faulty mitochondria. ${ }^{102}$ This leads to overall enhanced efficiency of mitochondria, and thus reduces oxidative stress and mutagenesis. Interestingly, severe caloric restriction also reduces basal metabolic rates, which is associated with decreased cancer frequency. ${ }^{103}$ Furthermore, high-fat diets are associated with tumorigenesis, ${ }^{104}$ probably due to the increase in monoacylglycerol lipase (MAGL). It is correlated with tumor aggressiveness and invasiveness, possibly through free-fatty acid release. Interestingly, reducing MAGL through siRNA inhibits tumor growth and invasiveness. ${ }^{105}$

Moreover, there is an association between diabetes and cancer. Diabetic patients who receive metformin, an 
anti-diabetic drug that increases insulin sensitivity, have decreased incidence of cancer, ${ }^{106,107}$ and inhibits transformation. ${ }^{108}$

\section{2 | Inhibition of fatty acid oxidation}

Entry of pyruvate into the Krebs cycle, by pyruvate dehydrogenase (PDH), is inhibited in cancer cells. Reactivation of PDH activity by dichloroacetate stimulated cell death, in a number of xenografts and solid tumor cell lines. ${ }^{109}$ This supports the hypothesis that mitochondrial glucose oxidation might be incompatible with survival of cancer cells. Similarly, inhibition of fatty acid oxidation enhanced apoptosis, stimulated by several chemotherapeutic agents in cancer cell lines, ${ }^{110}$ in addition to palmitate-induced apoptosis in hematopoietic cells. ${ }^{111}$ Thus, mitochondrial metabolism of fatty acids is probably linked to cancer cell survival. With respect to the role of UCPs in the metabolic shift, linked to increased fatty acid and glutamine metabolism to favor glucose oxidation, developing therapies targeting these proteins might be promising. ${ }^{61}$ A possible approach is through mitochondrial uncoupling. Fatty acid oxidation has been linked to chemoresistance and mitochondrial uncoupling, it is tempting to speculate that Warburg's observations may indeed be the result of the preferential oxidation of fatty acids by cancer cell mitochondria. ${ }^{112}$ Recent investigations into the mechanisms that underlie the Warburg effect suggest that: 1) mitochondrial uncoupling can promote aerobic glycolysis, 2) aerobic glycolysis occurs along with oxidative metabolism of non-glucose carbon sources, and 3) mitochondrial uncoupling occurs with resistance to chemotherapy. In summary, the combined effect of increased mitochondrial efficiency and reduced basal metabolic rates and free fatty acid release could possibly cut down mutagenic oxidative stress via caloric restriction, and might prove to be a very effective treatment for cancer along with chemotherapy in the future. ${ }^{113,114}$

\section{3 | EGFR tyrosine kinase inhibitors}

EGFR TKIs have a massive effect on aerobic glycolysis and have the ability to reactivate oxidative phosphorylation. As well as causing apoptosis and arrest of growth, EGFR TKIs might reactivate oxidative phosphorylation and, thereby, oxygen consumption in cancer cells, which will improve sensitivity to chemotherapy and radiotherapy. ${ }^{115}$ Moreover, decrease in lactate secretion might raise extracellular $\mathrm{pH}$ and thereby improve the microenvironment conditions of cancer cells and thus the response to therapy. ${ }^{116}$

\section{4 | 2-Deoxy-D-glucose}

2-Deoxy-D-glucose (2-DG) is a synthetic glucose analog where hydrogen replaces the C-2 hydroxyl group. It has been investigated comprehensively in basic and clinical studies since the 1950s. ${ }^{117}$ 2-DG has been recognized to deplete cellular energy, increase oxidative stress, interfere with $N$-linked glycosylation, and stimulate autophagy,. ${ }^{118,119}$ However, it is commonly believed that 2-DG prevents cancer by inhibiting glycolysis. 2-DG is also transported by GLUTs, thus competitively inhibits glucose uptake. ${ }^{117,120}$

\section{5 | Dichloroacetate}

Dichloroacetate (DCA) activates mitochondria in cancer cells. The mitochondrial pyruvate dehydrogenase (PDC) complex is located in the matrix. It catalyzes the aerobic oxidation of glucose, pyruvate, lactate and alanine to acetyl CoA, which is the rate-limiting step in glycolysis. Acetyl CoA is a substrate in the TCA cycle. Thereby, PDC is the main facilitator of oxidative phosphorylation and is thereby very important in cellular energetics. ${ }^{121,122}$ DCA is a structural analog of pyruvate that enhances PDC by inhibiting pyruvate dehydrogenase kinase (PDK), ${ }^{123}$ thus maintaining the unphosphorylated form of PDC. ${ }^{122}$ Furthermore, DCA enhances PDC activity by inhibiting its turnover. ${ }^{124}$

\section{6 | 3-Bromopyruvate}

3-Bromopyruvate (3-BP) reduces glycolysis by inhibiting hexokinase $\mathrm{II}^{125}$ and blocking ATP production. ${ }^{126}$ Moreover, it dephosphorylates Bcl-2-associated death promoter protein (BAD) at Ser112: a pro-apoptotic protein that regulates glycolysis and apoptosis. ${ }^{127}$ Subsequently, $\mathrm{BAX}$, a protein essential for BAD, is removed and localized to the mitochondria, which alters the permeability of the mitochondrial membrane. Thus, it causes the discharge of cytochrome $c$ and, thereby, cell death. ${ }^{127}$ In addition, 3-BP decreases GAPDH activity through pyruvate, bringing about the anti-glycolytic and thus anticancer effects. ${ }^{128}$

\section{7 | The S1P/S1PR3 axis}

G-protein-coupled receptors (GPCRs) are the major family of cell membrane receptors and thus might be promising for cancer therapy. A study aimed to discover the biological functions of sphingosine 1-phosphate receptor 
3 (S1PR3), one of the members of GPCRs family, as a target to treat osteosarcoma. ${ }^{129}$ Its results shed light on an unidentified role for the S1P/S1PR3 axis, for introducing the Warburg effect through activating the YAP/c-MYC/ PGAM1 pathway. Additionally, it revealed that TY52156, an S1PR3 antagonist, had synergistic inhibitory effects, when used with methotrexate, on cell growth in osteosarcoma. Therefore, targeting the S1P/S1PR3 axis might establish a new method to treat osteosarcoma.

\section{8 | Isocitrate dehydrogenase 2}

A study discovered the mechanism and function of wild-type Isocitrate dehydrogenase 2 (IDH2) in helping lung cancer growth. ${ }^{130}$ It reported that IDH2 is upregulated and is thus an indicator of poor survival in lung cancer and several other cancers. Targeting IDH2 with shRNA decreased the expression of HIF1 $\alpha$, reducing proliferation and growth of lung cancer cells. Treatment of lung cancer cells with AGI-6780, a small molecule inhibitor of IDH2, PX-478, an inhibitor of HIF1 $\alpha$, or cultivation with octyl- $\alpha-K G$ repressed lung cancer cell proliferation. In another setting, IDH inhibitors have been used in clinical trials and $\alpha$-KG salts have been used as therapeutic nutrition. ${ }^{131}$ IDH2 supports the Warburg effect and thus lung cancer cell growth, which is refereed by HIF1- $\alpha$ activation, followed by a decrease in $\alpha$-KG. Thus, IDH2 may be a novel target to treat lung cancer.

\section{9 | Alpha-enolase}

A-enolase (ENO1) is usually overexpressed in tumors and is thus a molecular target for immunotherapy. A study silenced ENO1 in human cancer cell lines and assessed its impact by proteomic, biochemical, and functional methods. ${ }^{132}$ ENO1 silencing increased reactive oxygen species that were mostly produced by the sorbitol and NADPH oxidase pathways, along with increasing autophagy and catabolic pathway adaptations, which together inhibit tumor cell growth. Inhibition of ENO1, either alone or in combination with other ENO1-related pathway, paves the way for potential therapeutic strategies.

\subsection{0 | Transcription factor SIX1}

A study showed that transcription factor SIX1 directly increases the expression of numerous glycolytic genes, thus supporting the Warburg effect and tumor growth, both in vitro and in vivo. ${ }^{133}$ SIX1 regulates glycolysis through the histone acetyltransferases HBO1 and AIB1. SIX1 mutation in cancer enhances its ability to boost aerobic glycolysis and tumor growth. Mir 548a-3p directly inhibits the glycolytic role of SIX1. MiR 548a-3p is downregulated in cancer and correlates with SIX1 inversely. Thus, it is good prognostic marker in breast cancer. So, the MiR 548a-3p/SIX1 axis strongly associates aerobic glycolysis to carcinogenesis and may possibly be an effective way to target cancer.

\subsection{1 | Monoclonal antibodies}

Monoclonal antibodies (mAbs) are a developing class of anticancer drugs, due to their ability to a tumor growth by direct targeting or immune modulation ${ }^{134,135}$ Therefore, understanding whether the activity and function of mAbs are impacted by tumor acidity might present possible strategies to improve clinical effectiveness and to overcome resistance. ${ }^{136}$ Other strategies utilizing monoclonal antibodies to treat cancer have been reported. ${ }^{137}$

\section{4 | SIGNIFICANCE AND CLOSING REMARKS}

Aerobic fermentation "Warburg effect" is a metabolic hallmark of most tumors. Discoveries of cell death pathways, UPR and the epigenetic regulation of gene expression by histones, chromatin and RNAs renewed interest in TWarburg and reverse Warburg effects. This review pointed out to the concepts regarding mitochondrial metabolism and the ER under stress. Also, it addressed how Warburg and reverse Warburg contribute to the basis for tumorigenesis. We emphasized on human investigations and the potential therapeutic modalities based on presearch findings.

\section{CONFLICT OF INTEREST}

All authors declare that they do not have any conflict of interest.

\section{AUTHORS' CONTRIBUTION}

Larry H. Bernstein, Dina Johar contributed to conception, Larry H. Bernstein, Dina Johar, Ahmed O. Elmehrath, Rania Khalil, Mostafa Elberry, Sami Zaki, Samy Shalabi contributed to data analysis, manuscript writing, and reviewing, Dina Johar responded to editorial reviews; Dina Johar provided the ENDNOTE X9 software. All authors approved manuscript submission for publication.

\section{DATA AVAILABILITY STATEMENT}

Enquiries about data can be directed to Dr. Dina Johar. 


\section{ORCID}

Dina Johar (10) https://orcid.org/0000-0003-0866-9959

Ahmed O. Elmehrath (10) https://orcid.org/0000-0002-2943-

4975

Rania M. Khalil (D) https://orcid.org/0000-0002-4970-2674

Mostafa H. Elberry (D) https://orcid.org/0000-0003-1212-

5137

Samy Zaky (i) https://orcid.org/0000-0003-4123-9221

Larry H. Bernstein (D) https://orcid.org/0000-0002-5274-

7742

\section{REFERENCES}

1. Warburg O. On the origin of cancer cells. Science. 1956; 123(3191):309-14.

2. Devlin TM. Textbook of biochemistry with clinical correlations. 6th ed. Hoboken, New Jersey: Wiley-Liss; 2006.p. 1208.

3. Ko YH, Delannoy M, Hullihen J, Chiu W, Pedersen PL. Mitochondrial ATP synthasome. Cristae-enriched membranes and a multiwell detergent screening assay yield dispersed single complexes containing the ATP synthase and carriers for Pi and ADP/ATP. J Biol Chem. 2003;278(14):12305-9.

4. Jiang B. Aerobic glycolysis and high level of lactate in cancer metabolism and microenvironment. Genes Dis. 2017;4(1): 25-7.

5. Boland ML, Chourasia AH, Macleod KF. Mitochondrial dysfunction in cancer. Front Oncol. 2013;3:292.

6. Yizhak K, Le Devedec SE, Rogkoti VM, Baenke F, de Boer VC, Frezza C, et al. A computational study of the Warburg effect identifies metabolic targets inhibiting cancer migration. Mol Syst Biol. 2014;10:744.

7. Board M, Humm S, Newsholme EA. Maximum activities of key enzymes of glycolysis, glutaminolysis, pentose phosphate pathway and tricarboxylic acid cycle in normal, neoplastic and suppressed cells. Biochem J. 1990;265(2):503-9.

8. Israelsen WJ, Vander Heiden MG. Pyruvate kinase: function, regulation and role in cancer. Semin Cell Dev Biol. 2015;43: 43-51.

9. Sellers K, Fox MP, Bousamra M, Slone SP, Higashi RM, Miller DM, et al. Pyruvate carboxylase is critical for nonsmall-cell lung cancer proliferation. J Clin Invest. 2015;125(2): 687-98.

10. Lunt SY, Muralidhar V, Hosios AM, Israelsen WJ, Gui DY, Newhouse L, et al. Pyruvate kinase isoform expression alters nucleotide synthesis to impact cell proliferation. Mol Cell. 2015;57(1):95-107.

11. de la Rosa V, Campos-Sandoval JA, Martin-Rufian M, Cardona C, Mates JM, Segura JA, et al. A novel glutaminase isoform in mammalian tissues. Neurochem Int. 2009;55(1-3):76-84.

12. Pavlides S, Whitaker-Menezes D, Castello-Cros R, Flomenberg N, Witkiewicz AK, Frank PG, et al. The reverse Warburg effect: aerobic glycolysis in cancer associated fibroblasts and the tumor stroma. Cell Cycle. 2009;8(23):3984-4001.

13. Yu I, Lu M, Jia D, Ma J, Ben-Jacob E, Levine H, et al. Modeling the genetic regulation of cancer metabolism: interplay between glycolysis and oxidative phosphorylation. Cancer Res. 2017;77(7):1564-74.

14. Wu HQ, Cheng ML, Lai JM, Wu HH, Chen MC, Liu WH, et al. Flux balance analysis predicts Warburg-like effects of mouse hepatocyte deficient in miR-122a. PLoS Comput Biol. 2017;13:e1005618.

15. Allen AE, Locasale JW. Glucose metabolism in cancer: the Saga of pyruvate kinase continues. Cancer Cell. 2018;33(3): 337-9.

16. Ochoa-Ruiz E, Diaz-Ruiz R. Anaplerosis in cancer: another step beyond the Warburg effect. Am J Mol Biol. 2012;02: 291-303.

17. Diaz-Ruiz R, Uribe-Carvajal S, Devin A, Rigoulet M. Tumor cell energy metabolism and its common features with yeast metabolism. Biochim Biophys Acta. 2009;1796(2):252-65.

18. Bramwell M, Humm S. Variations in the relative amounts of biotin-containing enzymes present in both tumorigenic and non-tumorigenic hybrid cells and other cell lines. Biochimica et Biophysica Acta (BBA)-molecular basis of disease. 1992; 1139(1--2):115-21.

19. Lee SY, Jeon HM, Ju MK, Kim CH, Yoon G, Han SI, et al. Wnt/Snail signaling regulates cytochrome $\mathrm{C}$ oxidase and glucose metabolism. Cancer Res. 2012;72(14):3607-17.

20. DeBerardinis R, Mancuso A, Daikhin E, Nissim I, Yudkoff M, Wehrli S, et al. Beyond aerobic glycolysis: transformed cells can engage in glutamine metabolism that exceeds the requirements for protein and nucleotide synthesis. Proc Natl Acad Sci. 2007;104:19345-50.

21. Hershko C. Control of disease by selective iron depletion: a novel therapeutic strategy utilizing iron chelators. Clin Haematol. 1994;7(4):965-1000.

22. Thelander L, Graslund A, Thelander M. Continual presence of oxygen and iron required for mammalian ribonucleotide reduction: possible regulation mechanism. Biochem Biophys Res Commun. 1983;110:859-65.

23. Thelander M, Graslund A, Thelander L. Subunit M2 of mammalian ribonucleotide reductase. Characterization of a homogeneous protein isolated from M2-overproducing mouse cells. J Biol Chem. 1985;260(5):2737-41.

24. Elliott RL, Head JF. Cancer: tumor iron metabolism, mitochondrial dysfunction and tumor immunosuppression; "a tight partnership-was Warburg correct?". J Cancer Ther. 2012;3(04):278-311.

25. Richardson DR, Lane DJ, Becker EM, Huang ML-H, Whitnall M, Rahmanto YS, et al. Mitochondrial iron trafficking and the integration of iron metabolism between the mitochondrion and cytosol. Proc Natl Acad Sci. 2010;107(24): 10775-82.

26. Ponizovskiy $\mathrm{M}$. The detailed description mechanisms of the herbs extracts operations in the new method cancer disease treatment via rearrangement of metabolism from pathologic development into normal development. J Clin Trials. 2012;2:2167.

27. Matoba S, Kang JG, Patino WD, Wragg A, Boehm M, Gavrilova O, et al. p53 regulates mitochondrial respiration. Science. 2006;312(5780):1650-3.

28. Sen N, Satija YK, Das S. p53 and metabolism: old player in a new game. Transcription. 2012;3:119-23.

29. San-Millán I, Brooks GA. Reexamining cancer metabolism: lactate production for carcinogenesis could be the purpose and explanation of the Warburg effect. Carcinogenesis. 2017; 38(2):119-33.

30. Ponisovskiy M. Warburg effect mechanism as the target for theoretical substantiation of a new potential cancer treatment. Crit Rev Eukaryot Gene Expr. 2011;21(1):13-28. 
31. Xiong L, Edwards CK, Zhou L. The biological function and clinical utilization of CD147 in human diseases: a review of the current scientific literature. Int J Mol Sci. 2014;15(10): 17411-41.

32. Tang X, Guo N, Xu L, Gou X, Mi M. CD147/EMMPRIN: an effective therapeutic target for hepatocellular carcinoma. J Drug Target. 2013;21(3):224-31.

33. Le Floch R, Chiche J, Marchiq I, Naiken T, Ilc K, Murray CM, et al. CD147 subunit of lactate/H+ symporters MCT1 and hypoxia-inducible MCT4 is critical for energetics and growth of glycolytic tumors. Proc Natl Acad Sci. 2011;108(40): 16663-8.

34. Semenza GL. HIF-1 mediates the Warburg effect in clear cell renal carcinoma. J Bioenerg Biomembr. 2007;39(3):231-4.

35. Semenza GL. HIF-1: upstream and downstream of cancer metabolism. Curr Opin Genet Dev. 2010;20(1):51-6.

36. Semenza GL. Hypoxia-inducible factors: mediators of cancer progression and targets for cancer therapy. Trends Pharmacol Sci. 2012;33(4):207-14.

37. Semenza GL. Hypoxia-inducible factors: coupling glucose metabolism and redox regulation with induction of the breast cancer stem cell phenotype. EMBO J. 2017;36(3):252-9.

38. Semenza GL, Roth PH, Fang HM, Wang GL. Transcriptional regulation of genes encoding glycolytic enzymes by hypoxiainducible factor 1. J Biol Chem. 1994;269(38):23757-63.

39. Shi DY, Xie FZ, Zhai C, Stern JS, Liu Y, Liu SL. The role of cellular oxidative stress in regulating glycolysis energy metabolism in hepatoma cells. Mol Cancer. 2009;8(1):32.

40. Johar DR, Bernstein LH. Biomarkers of stress-mediated metabolic deregulation in diabetes mellitus. Diabetes Res Clin Pract. 2017;126:222-9.

41. Zundel W, Schindler C, Haas-Kogan D, Koong A, Kaper F, Chen E, et al. Loss of PTEN facilitates HIF-1-mediated gene expression. Genes Dev. 2000;14(4):391-6.

42. Ravi R, Mookerjee B, Bhujwalla ZM, Sutter CH, Artemov D, Zeng Q, et al. Regulation of tumor angiogenesis by p53-induced degradation of hypoxia-inducible factor 1alpha. Genes Dev. 2000;14(1):34-44.

43. Ohh M. Ubiquitin pathway in VHL cancer syndrome. Neoplasia. 2006;8(8):623-9.

44. Dang C. The interplay between MYC and HIF in the Warburg effect. Ernst Schering Found Symp Proc. 2007;35-53.

45. Mazure NM, Chen EY, Laderoute KR, Giaccia AJ. Induction of vascular endothelial growth factor by hypoxia is modulated by a phosphatidylinositol 3-kinase/Akt signaling pathway in Ha-ras-transformed cells through a hypoxia inducible factor-1 transcriptional element. Blood. 1997;90(9): 3322-31.

46. Majumder PK, Febbo PG, Bikoff R, Berger R, Xue Q, McMahon LM, et al. mTOR inhibition reverses Aktdependent prostate intraepithelial neoplasia through regulation of apoptotic and HIF-1-dependent pathways. Nat Med. 2004;10(6):594-601.

47. Selak MA, Armour SM, MacKenzie ED, Boulahbel $\mathrm{H}$, Watson DG, Mansfield KD, et al. Succinate links TCA cycle dysfunction to oncogenesis by inhibiting HIF-alpha prolyl hydroxylase. Cancer Cell. 2005;7(1):77-85.

48. Isaacs JS, Jung YJ, Mole DR, Lee S, Torres-Cabala C, Chung YL, et al. HIF overexpression correlates with biallelic loss of fumarate hydratase in renal cancer: novel role of fumarate in regulation of HIF stability. Cancer Cell. 2005;8(2): 143-53.

49. Lu H, Forbes RA, Verma A. Hypoxia-inducible factor 1 activation by aerobic glycolysis implicates the Warburg effect in carcinogenesis. J Biol Chem. 2002;277(26):23111-5.

50. Courtnay R, Ngo DC, Malik N, Ververis K, Tortorella SM, Karagiannis TC. Cancer metabolism and the Warburg effect: the role of HIF-1 and PI3K. Mol Biol Rep. 2015;42(4):841-51.

51. Le A, Cooper CR, Gouw AM, Dinavahi R, Maitra A, Deck LM, et al. Inhibition of lactate dehydrogenase A induces oxidative stress and inhibits tumor progression. Proc Natl Acad Sci U S A. 2010;107(5):2037-42.

52. Ullah MS, Davies AJ, Halestrap AP. The plasma membrane lactate transporter MCT4, but not MCT1, is up-regulated by hypoxia through a HIF-1 $\alpha$-dependent mechanism. J Biol Chem. 2006;281(14):9030-7.

53. Kim JW, Tchernyshyov I, Semenza GL, Dang CV. HIF1-mediated expression of pyruvate dehydrogenase kinase: a metabolic switch required for cellular adaptation to hypoxia. Cell Metab. 2006;3(3):177-85.

54. Kroemer G, Pouyssegur J. Tumor cell metabolism: cancer's Achilles' heel. Cancer Cell. 2008;13(6):472-82.

55. Zhang H, Gao P, Fukuda R, Kumar G, Krishnamachary B, Zeller KI, et al. HIF-1 inhibits mitochondrial biogenesis and cellular respiration in VHL-deficient renal cell carcinoma by repression of C-MYC activity. Cancer Cell. 2007;11(5): 407-20.

56. Dang CV, Kim J, Gao P, Yustein J. The interplay between MYC and HIF in cancer. Nat Rev Cancer. 2008;8(1):51-6.

57. Maynard MA, Ohh M. Von Hippel-Lindau tumor suppressor protein and hypoxia-inducible factor in kidney cancer. Am J Nephrol. 2004;24(1):1-13.

58. Cai, W., Yang, H. The structure and regulation of Cullin 2 based E3 ubiquitin ligases and their biological functions. Cell Div. 2016;11:7.

59. Lando D, Peet DJ, Whelan DA, Gorman JJ, Whitelaw ML. Asparagine hydroxylation of the HIF transactivation domain a hypoxic switch. Science. 2002;295(5556):858-61.

60. Lu H, Li X, Luo Z, Liu J, Fan Z. Cetuximab reverses the Warburg effect by inhibiting HIF-1-regulated LDH-A. Mol Cancer Ther. 2013;12(10):2187-99.

61. Samudio I, Fiegl M, Andreeff M. Mitochondrial uncoupling and the Warburg effect: molecular basis for the reprogramming of cancer cell metabolism. Cancer Res. 2009;69(6): 2163-6.

62. Baffy G, Derdak Z, Robson SC. Mitochondrial recoupling: a novel therapeutic strategy for cancer? Br J Cancer. 2011; 105(4):469-74.

63. Hughes J, Criscuolo F. Evolutionary history of the UCP gene family: gene duplication and selection. BMC Evol Biol. 2008; 8(1):306.

64. Donadelli M, Dando I, Dalla Pozza E, Palmieri M. Mitochondrial uncoupling protein 2 and pancreatic cancer: a new potential target therapy. World J Gastroenterol. 2015;21(11): 3232-8.

65. Baffy G. Mitochondrial uncoupling in cancer cells: liabilities and opportunities. Biochim Biophys Acta Bioenerg. 2017; 1858(8):655-64. 
66. Ayyasamy V, Owens KM, Desouki MM, Liang P, Bakin A, Thangaraj K, et al. Cellular model of Warburg effect identifies tumor promoting function of UCP2 in breast cancer and its suppression by genipin. PLoS One. 2011;6(9):e24792.

67. Derdak Z, Mark NM, Beldi G, Robson SC, Wands JR, Baffy G. The mitochondrial uncoupling protein-2 promotes chemoresistance in cancer cells. Cancer Res. 2008;68(8): 2813-9.

68. Dando I, Fiorini C, Pozza ED, Padroni C, Costanzo C, Palmieri M, et al. UCP2 inhibition triggers ROS-dependent nuclear translocation of GAPDH and autophagic cell death in pancreatic adenocarcinoma cells. Biochim Biophys Acta. 2013;1833(3):672-9.

69. Brandi J, Cecconi D, Cordani M, Torrens-Mas M, Pacchiana R, Dalla Pozza E, et al. The antioxidant uncoupling protein 2 stimulates hnRNPA2/B1, GLUT1 and PKM2 expression and sensitizes pancreas cancer cells to glycolysis inhibition. Free Radic Biol Med. 2016;101:305-16.

70. Sreedhar A, Petruska P, Miriyala S, Panchatcharam M, Zhao Y. UCP2 overexpression enhanced glycolysis via activation of PFKFB2 during skin cell transformation. Oncotarget. 2017;8(56):95504-15.

71. Kulawiec M, Safina A, Desouki MM, Still I, Matsui S, Bakin A, et al. Tumorigenic transformation of human breast epithelial cells induced by mitochondrial DNA depletion. Cancer Biol Ther. 2008;7(11):1732-43.

72. Bouillaud F. UCP2, not a physiologically relevant uncoupler but a glucose sparing switch impacting ROS production and glucose sensing. Biochim Biophys Acta (BBA)-Bioenergetics. 2009;1787(5):377-83.

73. Baffy G. Uncoupling protein-2 and cancer. Mitochondrion. 2010;10(3):243-52.

74. Vozza A, Parisi G, De Leonardis F, Lasorsa FM, Castegna A, Amorese D, et al. UCP2 transports C4 metabolites out of mitochondria, regulating glucose and glutamine oxidation. Proc Natl Acad Sci U S A. 2014;111(3):960-5.

75. Colell A, Green DR, Ricci JE. Novel roles for GAPDH in cell death and carcinogenesis. Cell Death Differ. 2009;16(12): 1573-81.

76. Cho YS, Lee JH, Jung K-H, Park J-W, Moon SH, Choe YS, et al. Molecular mechanism of 18F-FDG uptake reduction induced by genipin in T47D cancer cell and role of uncoupling protein-2 in cancer cell glucose metabolism. Nucl Med Biol. 2016;43(10):587-92.

77. Burgermeister E, Liscovitch M, Rocken C, Schmid RM, Ebert MP. Caveats of caveolin-1 in cancer progression. Cancer Lett. 2008;268(2):187-201.

78. Nwosu ZC, Ebert MP, Dooley S, Meyer C. Caveolin-1 in the regulation of cell metabolism: a cancer perspective. Mol Cancer. 2016;15(1):71.

79. Kawaraguchi Y, Horikawa YT, Murphy AN, Murray F, Miyanohara A, Ali SS, et al. Volatile anesthetics protect cancer cells against tumor necrosis factor-related apoptosisinducing ligand-induced apoptosis via caveolins. Anesthesiology. 2011;115(3):499-508.

80. Ha TK, Her NG, Lee MG, Ryu BK, Lee JH, Han J, et al. Caveolin-1 increases aerobic glycolysis in colorectal cancers by stimulating HMGA1-mediated GLUT3 transcription. Cancer Res. 2012;72(16):4097-109.
81. Tahir SA, Yang G, Goltsov A, Song KD, Ren C, Wang J, et al. Caveolin-1-LRP6 signaling module stimulates aerobic glycolysis in prostate cancer. Cancer Res. 2013;73(6): 1900-11.

82. Raikar LS, Vallejo J, Lloyd PG, Hardin CD. Overexpression of caveolin-1 results in increased plasma membrane targeting of glycolytic enzymes: the structural basis for a membrane associated metabolic compartment. J Cell Biochem. 2006; 98(4):861-71

83. Christofk $H$, Vander Heiden $M$, Harris $M$, Ramanathan A, Gerszten R, Wei R, et al. The M2 splice isoform of pyruvate kinase is important for cancer metabolism and tumor growth. Nature. 2008;452:230-3.

84. Hart PC, Ratti BA, Mao M, Ansenberger-Fricano K, Shajahan-Haq AN, Tyner AL, et al. Caveolin-1 regulates cancer cell metabolism via scavenging Nrf2 and suppressing MnSOD-driven glycolysis. Oncotarget. 2016; 7(1):308-22.

85. Sotgia F, Martinez-Outschoorn UE, Pavlides S, Howell A, Pestell RG, Lisanti MP. Understanding the Warburg effect and the prognostic value of stromal caveolin-1 as a marker of a lethal tumor microenvironment. Breast Cancer Res. 2011; 13(4):213.

86. Martinez-Outschoorn UE, Balliet RM, Rivadeneira DB, Chiavarina B, Pavlides S, Wang C, et al. Oxidative stress in cancer associated fibroblasts drives tumor-stroma co-evolution: a new paradigm for understanding tumor metabolism, the field effect and genomic instability in cancer cells. Cell Cycle. 2010;9(16):3256-76.

87. Tidwell TR, Soreide K, Hagland HR. Aging, metabolism, and cancer development: from Peto's paradox to the Warburg effect. Aging Dis. 2017;8(5):662-76.

88. Seyfried TN, Mukherjee P. Targeting energy metabolism in brain cancer: review and hypothesis. Nutr Metab (Lond). 2005;2(1):30.

89. Schwartz L, Seyfried T, Alfarouk KO, Moreira JDV, Fais S Out of Warburg effect: an effective cancer treatment targeting the tumor specific metabolism and dysregulated $\mathrm{pH}$. Semin Cancer Biol. 2017;134-138.

90. Xing F, Luan Y, Cai J, Wu S, Mai J, Gu J, et al. The antiWarburg effect elicited by the cAMP-PGC1 $\alpha$ pathway drives differentiation of glioblastoma cells into astrocytes. Cell Rep. 2017;18(2):468-81.

91. Li S, Gao J, Zhuang X, Zhao C, Hou X, Xing X, et al. Cyclin G2 inhibits the Warburg effect and tumour progression by suppressing LDHA phosphorylation in glioma. Int J Biol Sci. 2019;15(3):544-55.

92. Calvisi DF. CD147/Basigin: a Warburg oncogene in hepatocellular carcinoma? Chin J Cancer Res. 2016;28(3):377-9.

93. Hursting SD, Smith SM, Lashinger LM, Harvey AE, Perkins SN. Calories and carcinogenesis: lessons learned from 30 years of calorie restriction research. Carcinogenesis. 2010; 31(1):83-9.

94. Longo VD, Fontana L. Calorie restriction and cancer prevention: metabolic and molecular mechanisms. Trends Pharmacol Sci. 2010;31(2):89-98.

95. Kalaany NY, Sabatini DM. Tumours with PI3K activation are resistant to dietary restriction. Nature. 2009;458(7239): 725-31. 
96. Mihaylova MM, Shaw RJ. The AMPK signalling pathway coordinates cell growth, autophagy and metabolism. Nat Cell Biol. 2011;13(9):1016-23.

97. Blagosklonny M. Linking calorie restriction to longevity through sirtuins and autophagy: any role for TOR. Cell Death Dis. 2010;1:e12.

98. Morselli E, Maiuri M, Markaki M, Megalou E, Pasparaki A, Palikaras K, et al. Caloric restriction and resveratrol promote longevity through the Sirtuin-1-dependent induction of autophagy. Cell Death Dis. 2010;1(1):e10.

99. Dang CV. Links between metabolism and cancer. Genes Dev. 2012;26(9):877-90.

100. Rabinowitz JD, White E. Autophagy and metabolism. Science. 2010;330(6009):1344-8.

101. Rubinsztein DC, Marino G, Kroemer G. Autophagy and aging. Cell. 2011;146(5):682-95.

102. Youle RJ, Narendra DP. Mechanisms of mitophagy. Nat Rev Mol Cell Biol. 2011;12(1):9-14.

103. Colman RJ, Anderson RM, Johnson SC, Kastman EK, Kosmatka KJ, Beasley TM, et al. Caloric restriction delays disease onset and mortality in rhesus monkeys. Science. 2009; 325(5937):201-4.

104. Venkateswaran V, Klotz LH. Diet and prostate cancer: mechanisms of action and implications for chemoprevention. Nat Rev Urol. 2010;7(8):442-53.

105. Nomura DK, Long JZ, Niessen S, Hoover HS, Ng S, Cravatt BF. Monoacylglycerol lipase regulates a fatty acid network that promotes cancer pathogenesis. Cell. 2010;140(1): 49-61.

106. Bowker SL, Majumdar SR, Veugelers P, Johnson JA. Increased cancer-related mortality for patients with type 2 diabetes who use sulfonylureas or insulin. Diabetes Care. 2006; 29(2):254-8.

107. Evans JM, Donnelly LA, Emslie-Smith AM, Alessi DR, Morris AD. Metformin and reduced risk of cancer in diabetic patients. BMJ. 2005;330(7503):1304-5.

108. Nicklin P, Bergman P, Zhang B, Triantafellow E, Wang H, Nyfeler B, et al. Bidirectional transport of amino acids regulates mTOR and autophagy. Cell. 2009;136(3):521-34.

109. Bonnet S, Archer SL, Allalunis-Turner J, Haromy A, Beaulieu C, Thompson R, et al. A mitochondria-K+ channel axis is suppressed in cancer and its normalization promotes apoptosis and inhibits cancer growth. Cancer Cell. 2007;11(1): 37-51.

110. Hernlund E, Ihrlund LS, Khan O, Ates YO, Linder S, Panaretakis T, et al. Potentiation of chemotherapeutic drugs by energy metabolism inhibitors 2-deoxyglucose and etomoxir. Int J Cancer. 2008;123(2):476-83.

111. Paumen MB, Ishida $\mathrm{Y}$, Muramatsu $\mathrm{M}$, Yamamoto $\mathrm{M}$, Honjo T. Inhibition of carnitine palmitoyltransferase I augments sphingolipid synthesis and palmitate-induced apoptosis. J Biol Chem. 1997;272(6):3324-9.

112. I Samudieo MF, Andreeff M. Mitochondrial uncoupling and the Warburg effect: molecular basis for the reprogramming of cancer cell metabolism. Cancer Res. 2009;69:2163.

113. El Mjiyad N, Caro-Maldonado A, Ramirez-Peinado S, MunozPinedo C. Sugar-free approaches to cancer cell killing. Oncogene. 2011;30(3):253-64.
114. Lee C, Longo VD. Fasting vs dietary restriction in cellular protection and cancer treatment: from model organisms to patients. Oncogene. 2011;30(30):3305-16.

115. De Rosa V, Iommelli $\mathrm{F}$, Monti $\mathrm{M}$, Fonti $\mathrm{R}$, Votta $\mathrm{G}$, Stoppelli MP, et al. Reversal of Warburg effect and reactivation of oxidative phosphorylation by differential inhibition of EGFR signaling pathways in non-small cell lung cancer. Clin Cancer Res. 2015;21(22):5110-20.

116. Hsu PP, Sabatini DM. Cancer cell metabolism: Warburg and beyond. Cell. 2008;134(5):703-7.

117. Kurtoglu M, Maher JC, Lampidis TJ. Differential toxic mechanisms of 2-deoxy-D-glucose versus 2-fluorodeoxy-D-glucose in hypoxic and normoxic tumor cells. Antioxid Redox Signal. 2007;9(9):1383-90.

118. Bandugula VR, Rajendra PN. 2-Deoxy-D-glucose and ferulic acid modulates radiation response signaling in non-small cell lung cancer cells. Tumour Biol. 2013;34(1):251-9.

119. Giammarioli AM, Gambardella L, Barbati C, Pietraforte D, Tinari A, Alberton M, et al. Differential effects of the glycolysis inhibitor 2-deoxy-D-glucose on the activity of pro-apoptotic agents in metastatic melanoma cells, and induction of a cytoprotective autophagic response. Int J Cancer. 2012;131(4): E337-47.

120. Urakami K, Zangiacomi V, Yamaguchi K, Kusuhara M. Impact of 2-deoxy-D-glucose on the target metabolome profile of a human endometrial cancer cell line. Biomed Res. 2013; 34(5):221-9.

121. Zhou ZH, McCarthy DB, O'Connor CM, Reed LJ, Stoops JK. The remarkable structural and functional organization of the eukaryotic pyruvate dehydrogenase complexes. Proc Natl Acad Sci U S A. 2001;98(26):14802-7.

122. Bowker-Kinley MM, Davis WI, Wu P, Harris RA, Popov KM. Evidence for existence of tissue-specific regulation of the mammalian pyruvate dehydrogenase complex. Biochem J. 1998;329(1):191-6.

123. Roche T, Baker JC, Yan X, Hiromasa Y, Gong X, Peng T, et al. Distinct regulatory properties of pyruvate dehydrogenase kinase and phosphatase isoforms. Prog Nucleic Acid Res Mol Biol. 2001;70:33-75.

124. Tran Q, Lee H, Park J, Kim S-H, Park J. Targeting cancer metabolism-revisiting the Warburg effects. Toxicol Res. 2016; 32(3):177-93.

125. Geschwind JF, Georgiades CS, Ko YH, Pedersen PL. Recently elucidated energy catabolism pathways provide opportunities for novel treatments in hepatocellular carcinoma. Expert Rev Anticancer Ther. 2004;4(3):449-57.

126. Ko YH, Smith BL, Wang Y, Pomper MG, Rini DA, Torbenson MS, et al. Advanced cancers: eradication in all cases using 3-bromopyruvate therapy to deplete ATP. Biochem Biophys Res Commun. 2004;324(1): 269-75.

127. Danial N, Gramm CF, Scorrano L, Zhang CY, Krauss S, Ranger AM, et al. BAD and glucokinase reside in a mitochondrial complex that integrates glycolysis and apoptosis. Nature. 2003;424:952-6.

128. Ganapathy-Kanniappan S, Geschwind J-FH, Kunjithapatham R, Buijs M, Vossen JA, Tchernyshyov I, et al. Glyceraldehyde3-phosphate dehydrogenase (GAPDH) is pyruvylated during 
3-bromopyruvate mediated cancer cell death. Anticancer Res. 2009;29(12):4909-18.

129. Shen $\mathrm{Y}$, Zhao S, Wang S, Pan X, Zhang $\mathrm{Y}$, Xu J, et al. S1P/S1PR3 axis promotes aerobic glycolysis by YAP/cMYC/PGAM1 axis in osteosarcoma. EBioMedicine. 2019;40: 210-23.

130. Li J, He Y, Tan Z, Lu J, Li L, Song X, et al. Wild-type IDH2 promotes the Warburg effect and tumor growth through HIF1 $\alpha$ in lung cancer. Theranostics. 2018;8(15):4050-61.

131. Cynober LA. The use of $\alpha$-ketoglutarate salts in clinical nutrition and metabolic care. Curr Opin Clin Nutr Metabol Care. 1999;2(1):33-7.

132. Capello M, Ferri-Borgogno S, Riganti C, Chattaragada MS, Principe M, Roux C, et al. Targeting the Warburg effect in cancer cells through ENO1 knockdown rescues oxidative phosphorylation and induces growth arrest. Oncotarget. 2016;7(5):5598-612.

133. Li L, Liang Y, Kang L, Liu Y, Gao S, Chen S, et al. Transcriptional regulation of the Warburg effect in cancer by SIX1. Cancer Cell. 2018;33(3):368-85. e7.

134. Scott AM, Wolchok JD, Old LJ. Antibody therapy of cancer. Nat Rev Cancer. 2012;12(4):278-87.
135. Menon S, Shin S, Dy G. Advances in cancer immunotherapy in solid tumors. Cancers. 2016;8(12):106.

136. Huber V, Camisaschi C, Berzi A, Ferro S, Lugini L, Triulzi T, et al. Cancer acidity: an ultimate frontier of tumor immune escape and a novel target of immunomodulation. Semin Cancer Biol. 2017;43:74-89.

137. Booy EP, Johar D, Maddika S, Pirzada H, Sahib MM, Gehrke I, et al. Monoclonal and bispecific antibodies as novel therapeutics. Arch Immunol Ther Exp (Warsz). 2006;54(2): 85-101.

How to cite this article: Johar D, Elmehrath AO, Khalil RM, Elberry MH, Zaky S, Shalabi SA, et al. Protein networks linking Warburg and reverse Warburg effects to cancer cell metabolism. BioFactors. 2021;1-16. https://doi.org/10.1002/ biof. 1768 